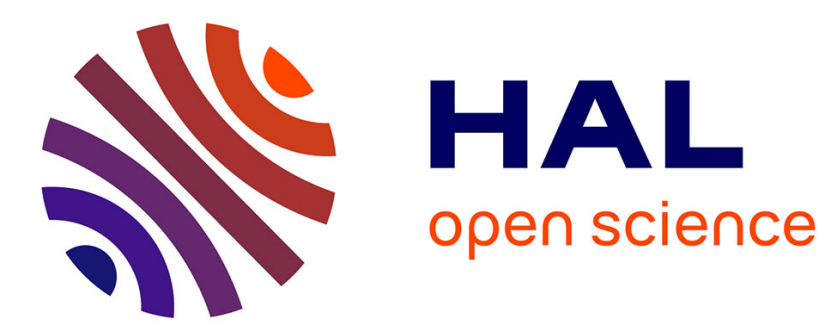

\title{
Réseau à trois dimensions de quadrupôles électriques élémentaires pour le confinement d'ions
}

\author{
F.G. Major
}

\section{To cite this version:}

F.G. Major. Réseau à trois dimensions de quadrupôles électriques élémentaires pour le confinement d'ions. Journal de Physique Lettres, 1977, 38 (12), pp.221-225. 10.1051/jphyslet:019770038012022100 . jpa-00231364

HAL Id: jpa-00231364 https://hal.science/jpa-00231364

Submitted on 1 Jan 1977

HAL is a multi-disciplinary open access archive for the deposit and dissemination of scientific research documents, whether they are published or not. The documents may come from teaching and research institutions in France or abroad, or from public or private research centers.
L'archive ouverte pluridisciplinaire HAL, est destinée au dépôt et à la diffusion de documents scientifiques de niveau recherche, publiés ou non, émanant des établissements d'enseignement et de recherche français ou étrangers, des laboratoires publics ou privés. 


\title{
RÉSEAU A TROIS DIMENSIONS DE QUADRUPÔLES ÉLECTRIQUES ÉLÉMENTAIRES POUR LE CONFINEMENT D'IONS
}

\author{
F. G. MAJOR $\left({ }^{*}\right)$ \\ University of the District of Columbia \\ Washington, D.C. 20008, U.S.A.
}

(Reçu le 24 mars 1977, accepté le 12 mai 1977)

\begin{abstract}
Résumé. - Nous proposons une méthode pour surmonter les limitations actuelles de la technique de stockage d'ions sous vide par des champs électromagnétiques. La méthode proposée utilise un réseau à trois dimensions de quadrupoles, chacun d'étendue microscopique, aux centres desquels les ions sont maintenus en suspension. Nous montrons que le nombre maximal d'ions confinés est augmenté, que l'énergie cinétique maximale des ions confinés est réduite, et que l'échange d'énergie entre les ions et le circuit extérieur est plus efficace dans cette méthode.
\end{abstract}

\begin{abstract}
We propose a method to overcome the present limitations of the technique of storing ions in vacuum by electromagnetic fields. The method proposed uses a three dimensional lattice of quadrupoles, each microscopic in size, at whose centers the ions are kept in suspension. We show that the maximum number of confined ions in increased, the maximum kinetic energy of the ions is reduced, and the exchange of energy between the ions and the outside circuit more efficient in this arrangement.
\end{abstract}

1. Introduction. $\rightarrow$ Les idées présentées ici sont destinées à surmonter les limitations actuelles de la technique de stockage d'ions dans le vide par des champs électromagnétiques, pour l'application à la spectroscopie à haute résolution. En particulier, nous proposons de remplacer la trappe utilisée couramment pour stocker des ions dans un espace étendu, que chaque ion parcourt sous l'action des forces de rappel créées par le champ, par une multiplicité de régions élémentaires de stockage, disposées dans un réseau à trois dimensions. Chaque région est d'étendue microscopique, et les ions sont maintenus en suspension en son centre. L'objet de cet article est de démontrer qu'un tel réseau possède des propriétés très intéressantes et de décrire une méthode pratique pour le réaliser dans une forme compatible avec les applications spectroscopiques.

Depuis quelques années, deux configurations de champ sont utilisées pour isoler des ions afin que leur spectre ne soit pas perturbé par des chocs avec d'autres particules ou des parois matérielles. L'une d'elles est composée d'un champ électrique à haute fréquence possédant une symétrie quadrupolaire [1], et a été utilisée pour l'étude des spectres hertziens de $\mathrm{He}^{+}$[2], $\mathrm{H}_{2}^{+}$[3], $\mathrm{Hg}^{+}[4], \mathrm{Ba}^{+}$[5] ainsi que pour des appli-

(*) Visiteur au Laboratoire de l'Horloge Atomique, Université Paris-Sud, 91405 Orsay, France, été 1976. cations dans le domaine de la spectrométrie de masse [6]. L'autre configuration, dite de Penning [7] réalise le confinement axial par un champ électrostatique quadrupolaire, tandis que la stabilité radiale est assurée par un champ magnétique homogène le long de l'axe de symétrie. Il est connu que ces deux configurations sont remarquablement efficaces au point de vue de l'isolement des ions. Mais le domaine de leurs applications est limité par les points faibles suivants :

1. Le nombre d'ions que l'on peut confiner est faible.

2. L'énergie cinétique est répartie sur un grand domaine.

3. La région de confinement est grande par rapport à la longueur d'onde du rayonnement induisant les transitions quantiques.

Nous présentons une analyse simple des lois gouvernant les variations de ces caractéristiques en fonction des dimensions de la région de confinement, et nous montrons que les limitations signalées précédemment sont surmontées en réduisant ces dimensions et en remplissant l'espace initial par un grand nombre de petites cellules de confinement.

2. Nombre maximal d'ions. - Considérons d'abord la loi de similitude gouvernant le nombre maximal d'ions que l'on peut confiner en même temps, en fonction des dimensions de la région de confinement. 
On peut raisonnablement supposer que ce nombre est déterminé par la répulsion électrostatique entre les ions, et que la perturbation créée par cette interaction répulsive est faible vis-à-vis du champ de confinement. Soit $\varphi_{\mathrm{s}}$ le potentiel moyen engendré à la position d'un ion donné par tous les autres ions. La valeur de $\varphi_{\mathrm{s}}$ est obtenue à l'aide de l'intégrale bien connue suivante :

$$
\varphi_{\mathbf{s}}(\mathbf{r})=e \int \frac{n\left(\mathbf{r}^{\prime}\right) \mathrm{d}^{3} \mathbf{r}^{\prime}}{\left|\mathbf{r}-\mathbf{r}^{\prime}\right|}
$$

Les résultats expérimentaux montrent qu'en réalité $\varphi_{\mathrm{s}}$ n'approche qu'à deux ordres de grandeur près la profondeur du potentiel de confinement. Un modèle théorique [8], simple et stationnaire, n'est donc pas suffisant. Une analyse plus détaillée des propriétés dynamiques de l'interaction entre les ions et le champ de confinement est nécessaire. Il faut admettre la possibilité d'une répartition de la densité des ions à la fois inhomogène et non-stationnaire. Il s'agit alors d'analyser le problème dans le cadre de l'équation de Boltzmann qui tient compte de l'interaction électrostatique entre les ions, mais qui néglige les chocs (équation de Vlasov [9]). Cette équation a la forme suivante :

$$
\begin{aligned}
\frac{\partial f}{\partial t}+\mathbf{v} \cdot \frac{\partial f}{\partial \mathbf{r}}+\left(\omega_{0}^{2} \mathbf{r}\right. & -\frac{e^{2}}{m} \nabla \times \\
& \left.\times \int \frac{f\left(\mathbf{r}^{\prime}\right) \mathrm{d}^{3} \mathbf{r}^{\prime} \mathrm{d}^{3} \mathbf{v}}{\left|\mathbf{r}-\mathbf{r}^{\prime}\right|}\right) \cdot \frac{\partial f}{\partial \mathbf{v}}=0 .
\end{aligned}
$$

Introduisons les substitutions suivantes :

$$
\tau=\omega_{0} t, \quad \boldsymbol{\rho}=\frac{\mathbf{r}}{r_{0}}, \quad \boldsymbol{\beta}=\frac{\mathbf{v}}{\omega_{0} r_{0}}
$$

et

$$
\varphi=\frac{f}{\int f \mathrm{~d}^{3} \mathbf{r d}^{3} \mathbf{v}}
$$

L'équation est transformée en la forme suivante :

$$
\begin{aligned}
\frac{\partial \varphi}{\partial \tau}+\boldsymbol{\beta} \cdot \frac{\partial \varphi}{\partial \rho}+\left(\rho-\frac{e^{2} N}{m \omega_{0}^{2} r_{0}^{3}} \nabla_{\rho} \times\right. \\
\left.\times \int \frac{\varphi \mathrm{d}^{3} \rho \mathrm{d}^{3} \boldsymbol{\beta}}{\left|\rho-\boldsymbol{\rho}^{\prime}\right|}\right) \cdot \frac{\partial \varphi}{\partial \boldsymbol{\beta}}=0
\end{aligned}
$$

qui montre que les dimensions physiques sont toutes comprises dans le coefficient $e^{2} N / m \omega_{0}^{2} r_{0}^{3}$. La stabilité de la solution doit être dépendante uniquement de la valeur de ce coefficient, qui est alors la mesure véritable de l'influence relative du champ d'ions confinés. Sous la condition d'énergie constante, c'est-à-dire $m \omega_{0}^{2} r_{0}^{2}=\mathrm{Cte}$, on déduit le résultat suivant :

$$
e^{2} N_{\max }=2(A) E r_{0}
$$

où $A$ est une constante critique de l'équation, $E$ est l'énergie et $r_{0}$ est le rayon de la région de confinement.

La variation linéaire du nombre $N_{\max }$ en fonction du rayjon de la région de confinement a pour conséquence la possibilité d'une augmentation importante du nombre $N_{\max }$ En effet, considérons un espace donné ayant des dimensions linéaires $L$ et divisons le en $M^{3}$ sous-espaces de dimension $L / M$. Le nombre maximal d'ions est alors multiplié par $M^{2}$.

3. Energie cinétique maximale des ions. - L'énergie cinétique des ions confinés est déterminée par la profondeur du champ de potentiel effectif qui confine les ions. Il en résulte que le nombre maximal dérivé ci-dessus sous la condition de $E=$ Cte correspond alors à la condition de profondeur constante du potentiel.

Pour les applications spectroscopiques, la répartition de l'énergie cinétique des ions est souvent aussi intéressante que l'intensité du signal, c'est-à-dire, le nombre d'ions qui contribue au signal. Considérons alors la variation de l'énergie maximale en fonction des dimensions de la région de confinement en tenant constant le nombre total dans toutes les cellules élémentaires de confinement. Puisque nous supposons un espace total donné, la densité reste constante alors que cet espace est divisé en cellules élémentaires. Le nombre dans chaque cellule varie alors comme $r_{0}^{3}$, et il s'en suit que l'énergie varie comme $r_{0}^{2}$. Ce résultat signifie qu'une réduction importante de l'énergie cinétique peut être réalisée en réduisant les dimensions de la région de confinement.

Vérifions cette conclusion générale dans les cas particuliers de la configuration à haute fréquence de

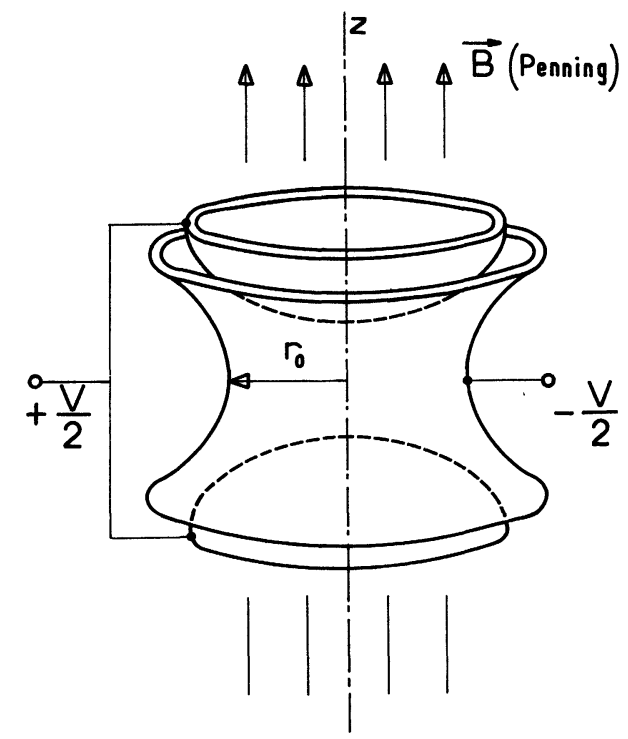

FIG. 1. - Géométrie des électrodes produisant le champ quadrupolaire pur. Dans la configuration de Penning, on a $V=U_{0}$, et dans celle de Paul $V=U_{0}+V_{0} \cos \Omega t$.

[Shape of the electrodes which create a pure quadrupole field. In the Penning configuration, one has $V=U_{0}$, and in the Paul configuration $V=U_{0}+V_{0} \cos \Omega t$.] 
Paul, et de la configuration statique de Penning. Supposons d'abord que tous les ions ont le même rapport $e / m$ et sont confinés dans un champ à haute fréquence quadrupolaire dérivé du potentiel suivant :

$$
\varphi=\left(U_{0}+V_{0} \cos \Omega t\right)\left(r^{2} / 2-z^{2}\right) / r_{0}^{2} .
$$

Les propriétés du mouvement des ions dans un tel champ ont déjà été discutées dans la littérature $[1,2,10]$ et ne seront pas répétées ici. Si la condition

$$
q=4 e V_{0} / m \Omega_{0}^{2} r_{0}^{2} \ll 1
$$

(dite condition adiabatique) est vérifiée, le mouvement est séparable en deux parties : le micromouvement de faible amplitude, à la fréquence $\Omega$, et le mouvement séculaire beaucoup plus lent, gouverné par un champ effectif dérivé du potentiel suivant :

$$
\varphi_{\mathrm{eff}}=\frac{q V_{0}}{16}\left(\frac{r^{2}}{r_{0}^{2}}+\frac{z^{2}}{\left(r_{0} / 2\right)^{2}}\right) .
$$

Il est évident que la quantité $q V_{0} / 16$ est la profondeur du champ effectif de potentiel, et qu'elle représente la limite de l'énergie cinétique des ions confinés. Les fréquences caractéristiques d'oscillation dans un tel champ sont $\left(e q V_{0} / 8 m r_{0}^{2}\right)^{1 / 2}$ et $\left(e q V_{0} / 2 m r_{0}^{2}\right)^{1 / 2}$ pour les coordonnées radiale et axiale respectivement. Quoique le mouvement oscillatoire ne soit pas isotrope dans ce cas puisqu'il possède deux fréquences différentes, on s'attend néanmoins à ce que les résultats obtenus ci-dessus restent valables. Supposons une densité moyenne globale donnée $(N / V)$, le nombre d'ions confinés dans une cellule est $(N / V) 2 \pi r_{0}^{3} / 3$, puisque l'espace occupé par les ions est limité par l'ellipsoïde

$$
\left(\frac{r^{2}}{r_{0}^{2}}+\frac{z^{2}}{\left(r_{0} / 2\right)^{2}}\right)=1
$$

dont le volume est $2 \pi r_{0}^{3} / 3$. En utilisant le résultat déduit de l'équation de Vlasov, on obtient pour l'énergie l'expression suivante :

$$
E=\frac{e^{2}(N / V) \pi}{3 A} r_{0}^{2} \quad(\text { Paul })
$$

Des raisonnements semblables s'appliquent aussi au cas de la configuration de Penning où le champ de confinement comprend la combinaison suivante (pour les ions positifs) :

$$
\varphi=-\frac{U_{0}}{r_{0}^{2}}\left(r^{2} / 2-z^{2}\right), \quad \mathbf{B}=B \mathbf{k} .
$$

Supposons que la condition usuelle

$$
e^{2} B^{2} / m^{2} \gg e U_{0} / m r_{0}^{2}
$$

est vérifiée. En ce cas, on peut supposer, sans perte de généralité, que le champ électrostatique détermine la limite du nombre d'ions confinés, et que la répartition de la densité des ions a la même symétrie d'ellipsoïdes confocales. Il en résulte que le volume de l'espace accessible aux ions est $4 \pi r_{0}^{2} z_{0}^{2} / 3$, et on a l'expression suivante pour l'énergie :

$$
E=\frac{\sqrt{2} 2 \pi e^{2}(N / V)}{3 A} r_{0}^{2} \quad \text { (Penning). }
$$

4. Couplage entre les ions et le circuit extérieur. Considérons ensuite le problème du couplage capacitif entre les ions oscillant dans le champ de confinement et une impédance extérieure à une température donnée [11]. La solution de ce problème détermine les conditions dans lesquelles il est possible pratiquement de refroidir les ions par le transfert efficace de leur énergie cinétique à un réservoir thermique extérieur.

On peut supposer avec une précision suffisante que le spectre du mouvement des ions a une fréquence unique, et qu'un ion oscillant à cette fréquence dans la direction de l'axe de symétrie induit un courant électrique

$$
e \frac{\mathrm{d} z}{\mathrm{~d} t} / 2 z_{0},
$$

où $2 z_{0}$ est la distance séparant les électrodes. Si la capacité $C$ formée par les électrodes est accordée avec une self de coefficient de qualité $Q$, une différence de potentiel instantanée

$$
\left(e Q / 2 \omega z_{0} C\right) \frac{\mathrm{d} z}{\mathrm{~d} t}
$$

apparaît entre les électrodes, et produit sur les ions une réaction dépendante de leur vitesse :

$$
F=\frac{e^{2}}{4 z_{0}^{2}} \frac{Q}{\omega C} \frac{\mathrm{d} z}{\mathrm{~d} t}
$$

qui correspond à un amortissemẹnt $\gamma$ de valeur

$$
\gamma=\frac{e^{2}}{4 m z_{0}^{2}} \frac{Q}{\omega C}
$$

Ce résultat montre que la constante d'amortissement varie comme l'inverse du carré des dimensions linéaires puisque l'impédance $Q / \omega C$ du circuit résonnant reste presque constante lorsque la fréquence est modifiée, par exemple dans le rapport 17 à 1 pour les selfs supraconductrices de $\mathrm{Nb}_{3} \mathrm{Sn}$, alliage qui conserve ses propriétés supraconductrices jusqu'à une température de $18 \mathrm{~K}$ [12].

5. Réalisation pratique - Une réalisation pratique de la configuration de confinement considérée ci-dessus, c'est-à-dire un réseau à trois dimensions engendré par la répétition d'un motif constitué par un quadrupole microscopique, devient évidente si l'on se 
souvient qu'un champ à symétrie quadrupolaire peut être créé avec une précision bien suffisante au voisinage d'une ouverture circulaire percée dans une lame conductrice au potentiel $\varphi$ comprise entre deux lames conductrices parallèles au potentiel zéro. On peut développer le potentiel au voisinage du centre d'une telle ouverture en série de Taylor :

$$
\varphi=\varphi_{0}+\frac{1}{2}\left(\frac{\partial^{2} \varphi}{\partial r^{2}}\right)_{0} r^{2}-\left(\frac{\partial^{2} \varphi}{\partial r^{2}}\right)_{0} z^{2}
$$

où l'on a utilisé

$$
\left(\frac{\partial \varphi}{\partial r}\right)_{0}=0, \quad\left(\frac{\partial \varphi}{\partial z}\right)_{0}=0, \quad \nabla^{2} \varphi=0 .
$$

On reconnaît dans cette expression la forme analytique caractéristique du champ quadrupolaire pur. On peut alors envisager un réseau constitué d'un grand nombre d'ouvertures disposées régulièrement dans chaque lame et d'un grand nombre de lames parallèles placées de telle façon que les centres des ouvertures soient alignés. La phase du potentiel à haute fréquence (ou la polarité, dans le cas de la trappe de Penning) est alternée sur chaque lame. Ainsi les plans à mi-distance entre les lames sont toujours au potentiel zéro sans qu'il soit nécessaire de les matérialiser.

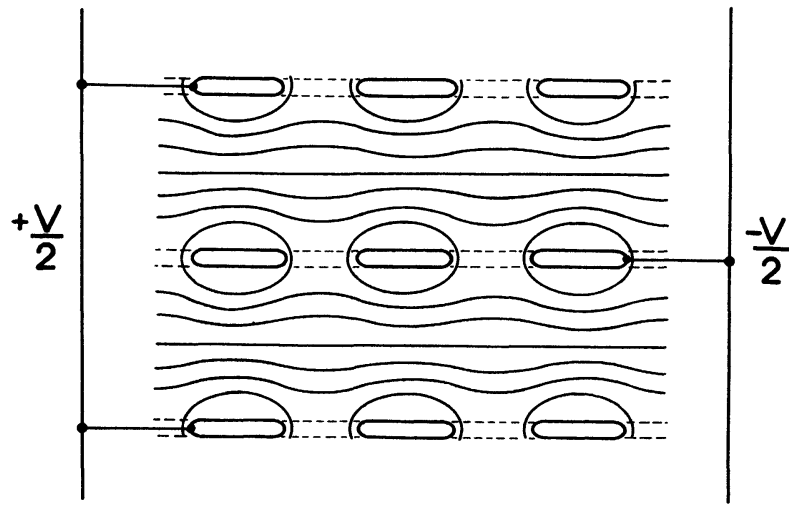

Fig. 2. - Un réseau à trois dimensions de quadrupoles électriques réalisés pratiquement par un grand nombre d'ouvertures disposées régulièrement dans des lames conductrices. Au voisinage de chaque ouverture, les équipotentielles ont même allure que dans une trappe classique, telle que représentée sur la figure 1.

[A three-dimensional array of electric quadrupoles can be realized by a large number of apertures regularly set in conducting strips. Near each apertures, the equipotential surfaces have the same as for a classical trap, as shown on figure 1.]

Les solutions détaillées pour le potentiel électrostatique créé par des systèmes d'électrodes à symétrie cylindrique ont été récemment publiées [13]. Les résultats sont exprimés en séries de Bessel-Fourier dont les coefficients sont obtenus par la résolution d'un système d'équations algébriques. La solution analytique suivante, valable sur l'axe de symétrie, a été donnée par Ollendorf [14] pour une ouverture unique séparant deux régions où les champs asymptotiques sont $E_{1}$ et $E_{2}$ :

$$
\begin{aligned}
\varphi(z)=\varphi_{1}-\frac{\left(E_{1}+E_{2}\right)}{2} z+\frac{\left(E_{1}-E_{2}\right)}{2} \times \\
\times\left[z \operatorname{arctg}\left(\frac{z}{R}\right)+R\right]
\end{aligned}
$$

où $R$ est le rayon de l'ouverture. Le cas particulier où $E_{1}=-E_{2}$, qui correspond à la disposition produisant un champ de symétrie quadrupolaire s'écrit :

$$
\varphi(z)=\varphi_{1}+E\left[z \operatorname{arctg}\left(\frac{z}{R}\right)+R\right] .
$$

Si les plans de potentiel zéro sont situés à la distance $d(d \gg R)$ du plan de l'ouverture, on a : $E=-2 \varphi_{1} / \pi d$, ce qui donne enfin :

$$
\varphi(z)=\varphi_{1}-\frac{2 \varphi_{1}}{\pi d}\left[z \operatorname{arctg}\left(\frac{z}{R}\right)+R\right]
$$

En utilisant le fait que $\varphi(z)$ est une solution de l'équation de Laplace, on peut démontrer qu'au voisinage de l'axe, la dépendance radiale de $\varphi$ révèle un minimum sur l'axe. Le premier terme dans le développement de $\varphi$ en série de Taylor est alors en $r^{2}$. Cette dernière propriété peut avoir une importance critique pour le remplissage des cellules de confinement par des ions. On peut, par exemple, créer les ions au dehors du système de confinement lui-même et les laisser dériver le long des lignes des centres des ouvertures. Naturellement, il est nécessaire d'extraire de l'énergie cinétique des ions pour stabiliser leur confinement aux centres des cellules élémentaires. L'échange de charge avec des atomes neutres, ou des chocs inélastiques avec d'autres particules peuvent être utilisés à cette fin.

Bien que le dispositif expérimental décrit plus haut suppose des ouvertures circulaires et des lames planes, la seule exigence critique est que la structure utilisée crée un potentiel électrique qui présente un grand nombre de points où il est localement minimum. Par exemple, un grillage fabriqué par expansion d'une feuille métallique pourrait convenir. Une possibilité spéculative, qui dans un espace de quelques centimètres cubes pourrait contenir de l'ordre de $10^{9}$ régions élémentaires, serait une structure réalisée par les techniques de dépôts de couches minces sous vide.

Remerciements. - L'auteur tient à exprimer sa profonde gratitude pour l'hospitalité qui lui a été offerte au Laboratoire de l'Horloge Atomique, et spécialement à MM. C. Audoin et J. L. Duchêne pour leur aide dans la préparation du manuscrit. 


\section{Bibliographie}

[1] Paul, W., Osberghaus, O. et Fischer, E., Forschungsber. Wirtsch. u. Verkehrsministeriums Nordrhein-Westfalen no 415 (1958).

FISCHER E., Z. Phys. 156 (1969) 1.

[2] Major, F. G. et Dehmelt, H. G., Phys. Rev. 170 (1968) 91. Voir aussi

Dehmelt, H. G. dans Adv. At. Mol. Phys. rédacteur Bates D. R., 5 (Academic Press, Inc New York) 1969, 109.

[3] Jefrerts, K. B., Phys. Rev. Lett. 20 (1968) 39. Voir aussi DeHMELT, H. G. ref. [2].

[4] Major, H. G. et Werth, G., Phys. Rev. Lett. 30 (1973) 1155

[5] Duchene, J. L., Audoin, C. et Schermann, J. P., C.R. Hebd. Séan. Acad. Sci. 107B (1976)

IFFLANDER, R. et WERTH, G., soumis à Metrologia (1976).

[6] Rettinghaus, G., Thèse de Doctorat, Physikalisches Institut, Bonn (1965).
[7] Penning, F. M., Physica 4 (1937) 71.

PIERCE, J. R., Theory and Design of Electron Beams (Van Nostrand, Princeton N.J.) 1949.

[8] Dehimel, H. G., Adv. At. Mol. Phys. 3 (1967) 55 and 5 (1969) 120.

[9] Voir par exemple WeIBet, E. S., Phys. Fluids 3 (1960) 399.

[10] Benilan, M. N. et Audoin, C., Int. J. Mass Spectrom. Ion Phys. 11 (1973) 421.

[11] Wineland, D. J. and Dehmelt, H. G., J. Appl. Phys. 46 (1975) 919.

[12] Vig, J. R. et Gixow, E., Proc. IEEE (USA) 61 (1973) 122.

[13] Wittels, N. D. et Jacobsen, E. H., J. Appl. Phys. 47 (1976) 2716.

[14] OllendoRf, F., Die Potentielfelder der Elektrontechnik (Springer Verlag, Berlin) 1932. 